\title{
VARIABILIDADE VERTICAL DE ESTRUTURAS COERENTES NA CAMADA LIMITE CONVECTIVA DA AMAZÔNIA
}

\author{
Cléo Q. Dias Jr. ${ }^{1}$, Leonardo D. A. Sá ${ }^{2}$, Edson P. Marques Filho ${ }^{3}$, Antonio O. Manzi ${ }^{1}$, Ivonne \\ Trebs $^{4}$, Jan Winderlich ${ }^{4}$ \\ ${ }^{1}$ Instituto Nacional de Pesquisas da Amazônia/ IFPA - Bragança - PA \\ ${ }^{2}$ Instituto Nacional de Pesquisas Espaciais/Belém, PA \\ ${ }^{3}$ Universidade Federal do Rio de Janeiro \\ ${ }^{4}$ Biogeochemistry Department Max Plank Institute for Chemistry, Germany. \\ cleo.quaresma@inpa.gov.br
}

\section{RESUMO}

Neste trabalho, foram detectadas as escalas temporais das estruturas coerentes (ECs) em dois níveis acima da reserva florestal do Uatumã, Amazonas. Os dados observacionais utilizados foram coletados na torre micrometeorológica de $81 \mathrm{~m}$ instalada na área de estudo do projeto ATTO. As ECs detectadas a uma altura de $41 \mathrm{~m}$ apresentaram escala temporal menor que as observadas em $81 \mathrm{~m}$.

\begin{abstract}
In this work were detected temporal scales of coherent structures at two levels above the forest reserve Uatumã, Amazonas. The observational data used were collected in micrometeorological tower of $81 \mathrm{~m}$ installed in the project study area ATTO. The coherent structures detected at height of $41 \mathrm{~m}$ presented time scale shorter than those observed in $81 \mathrm{~m}$.
\end{abstract}

\section{INTRODUÇÃO}

Nas últimas décadas, o conhecimento da turbulência avançou a ponto de reconhecer a importância das ECs na organização do escoamento turbulento da camada limite atmosférica (CLA). Essas estruturas manifestam-se, intermitentemente, como grandes desvios da média em séries temporais de medidas turbulentas e são reconhecidamente fundamentais na transferência de momento e escalares entre a vegetação e a atmosfera.

Recentemente, Dias Júnior et al. (2013) mostraram que a duração das ECs em condições diurnas, logo acima da copa florestal, está correlacionada com a altura do ponto de inflexão do perfil vertical da velocidade do vento. Outro aspecto interessante é o processo de coalescência que as estruturas térmicas sofrem com o aumento da altitude (Hellsten e Zilitinkevich, 2013).

Neste trabalho, efetuou-se estudo acerca da estrutura da turbulência atmosférica, sob condições instáveis, acima da floresta de Uatumã na Amazônia central, tendo por base as informações coletadas em torre alta de observação em dois níveis verticais, $41 \mathrm{~m}$ e $81 \mathrm{~m}$ acima do solo.

\section{MATERIAL E MÉTODOS}

As séries temporais de 30 minutos da velocidade vertical do vento(w), temperatura (T), umidade (q) e concentração de gás carbônico $\left(\mathrm{CO}_{2}\right)$, foram coletados por instrumentos de resposta rápida $(10 \mathrm{~Hz})$, à 13h30min hora local, no dia Juliano 185 de 2012 . A metodologia de detecção das ECs utilizada foi proposta por Thomas e Foken (2005). Para extrair informações acerca das ECs usou-se a função wavelet complexa de Morlet. 
A duração característica de uma estrutura coerente, $D_{e}$, foi definida a partir da duração do evento correspondente ao primeiro máximo no espectro de variância wavelet.

\section{RESULTADOS E DISCUSSÃO}

As Figuras 1a e 1b mostram as variâncias wavelets, normalizadas pelo seu valor máximo, calculadas em função da duração do evento para as alturas de $81 \mathrm{~m}$ e $42 \mathrm{~m}$, respectivamente. Observa-se que em $81 \mathrm{~m}, w$ exibe dois máximos, o primeiro localizado em $21.5 \mathrm{~s}$ e o segundo, mais pronunciado, em 58.34s. Os primeiros máximos dos três escalares $\left(T, \mathrm{CO}_{2}\right.$ e $\left.q\right)$ são coincidentes em torno de $52 \mathrm{~s}$ e se aproximam do segundo máximo de $w$. Desta forma, o valor de 58.34s foi escolhido como definição de De para $w$, uma vez que EC é definida como uma parcela de fluido que apresenta uma distinta relação de fase entre as propriedades do escoamento e suas constituintes (Blackwelder e Kaplan, 1976). Este resultado pode ser explicado pelos espectros dos escalares que apresentam maior similaridade com os espectros da velocidade horizontal comparativamente a velocidade vertical nas baixas frequências. Portanto, o transporte por processos de baixa frequência, tal como as ECs, está diretamente relacionado ao transporte horizontal. Para os dados correspondentes a altura de $41 \mathrm{~m}$, o espectro de variância de $w$, exibe o primeiro máximo em torno de 25s. Este valor é praticamente igual aos picos espectrais dos escalares, que ficou em torno de $23 \mathrm{~s}$.

a)

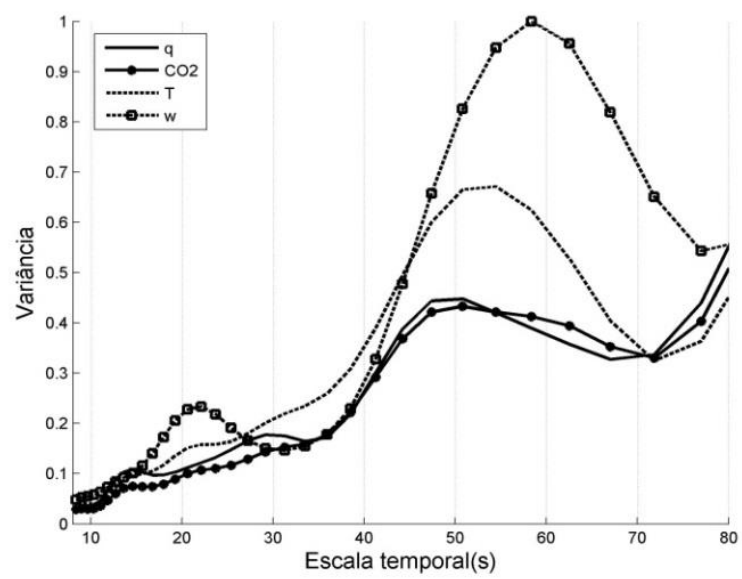

b)

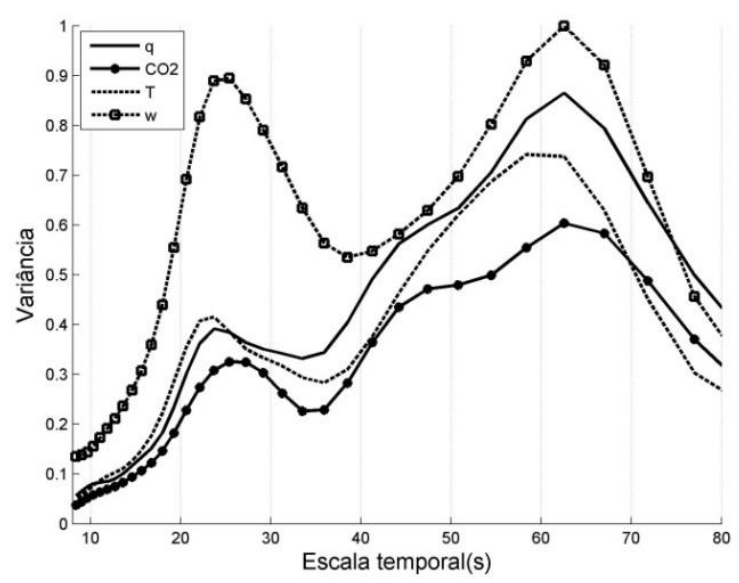


Figura 1: Variância por escala dos coeficientes wavelets da temperatura, velocidade vertical do vento, umidade e $\mathrm{CO}_{2}$ calculados para o dia juliano 185 do ano de 2012 as 13:30, hora local, nas alturas de: a) $81 \mathrm{~m}$ e b) $41 \mathrm{~m}$.

\section{CONCLUSÃO}

Neste estudo, as ECs apresentaram uma diminuição na duração à medida que se aproximaram do dossel florestal. Este fato pode estar relacionado à maior dissipação de energia próximo da superfície. Além disso, as ECs observadas em alturas mais distantes do dossel apresentaram uma escala temporal maior, devido, possivelmente, à coalescência dos vórtices turbulentos.

\section{AGRADECIMENTOS}

Este trabalho é parte do projeto LBA apoiado pela FAPEAM e pela UEA, com suporte financeiro do INPA do Max Plank Society. Os autores agradecem a CAPES pela bolsa de doutorado, e ao CNPq pelas bolsas de produtividade em pesquisa (processos $\mathrm{n}^{\circ}$ $303.728 / 2010-8$ e no $308.597 / 2012-5$ ).

\section{BIBLIOGRAFIA}

BLACKWELDER, R. F.; KAPLAN, R. E.On the wall structure of the turbulent boundary layer. Journal of Fluid Mechanics, v. 76, n. 1, p. 89-112, 1976.

DIAS JÚNIOR, C. Q. et al. Coherent structures detected in the unstable atmospheric surface layer above the Amazon forest. Journal of Wind Engineering and Industrial Aerodynamics, v. 115, p. 1-8, 2013.

THOMAS, Chr; FOKEN, Th. Detection of long-term coherent exchange over spruce forest using wavelet analysis. Theoretical and Applied Climatology, v. 80, n. 2-4, p. 91-104, 2005.

HELLSTEN, Antti; ZILITINKEVICH, Sergej. Role of Convective Structures and Background Turbulence in the Dry Convective Boundary Layer. Boundary-Layer Meteorology, p. 1-31, 2013. 